\title{
FIELD GALAXY EVOLUTION STUDIES WITH AN OPTICAL MULTICOLOR DEEP-SKY SURVEY
}

Charles T. Liu (U. of Arizona), Richard F. Green (NOAO),

Patrick B. Hall (U. of Arizona) and Patrick S. Osmer (Ohio State U.)

We are investigating field galaxy evolution with the optical multiband survey of Osmer et al. (1995, in preparation; Hall et al. 1992, Bull. A.A.S., 24, 1136), which covers some 3000 sq. arcmin of sky with CCD photometry in six broad-band filters from $3000-10000 \AA$, complete down to $R \sim 23$. The sample contains some 9000 cataloged galaxies, of which 7000 have data in at least three colors.

Our approach has been to identify both the star formation rate (SFR) and redshift of the field galaxies in the survey from their multicolors. Model galaxy colors in the $\mathrm{U} / \mathrm{B} / \mathrm{V} / \mathrm{R} / \mathrm{I}(7500 \AA) / \mathrm{I}(8600 \AA)$ passbands were produced using spectral energy distributions of typical E/S0, Sbc, Scd and Irr galaxies created with the spectrophotometric data of Kennicutt (1992, ApJS, 79, 255) and Coleman et al. (1980, ApJS, 43, 393). In the spirit of Koo $(1985, \mathrm{AJ}, 90,418)$ and others, it is possible to distinguish the spectroscopic Hubble types and redshifts of each galaxy type using different projections in multi-dimensional color space (Fig. 1). Since our I-band filters are relatively narrow (1000 $\AA$ FWHM), we have additional leverage for high-redshift objects near $z \sim 1$.

We have applied these models to a complete subsample of 438 red galaxies from the sample. Red galaxies were selected for several reasons: (1) the $4000 \mathrm{~A}$ break is generally strong, aiding in more accurate redshift determinations; (2) degeneracies caused by such factors as reddening are less of an obstacle; (3) luminosity evolution in the red galaxy population can be well quantified using no-evolution models such as these. Our preliminary analysis appears to confirm the effectiveness of our technique for redshift identifications of these data. With the overall galaxy sample, we hope to compute luminosity functions of faint field galaxies with respect to magnitude, redshift and spectral type. With that information, we will estimate the total SFR in field galaxies with respect to cosmic time and the fractional SFR contributions from galaxy sub-types.
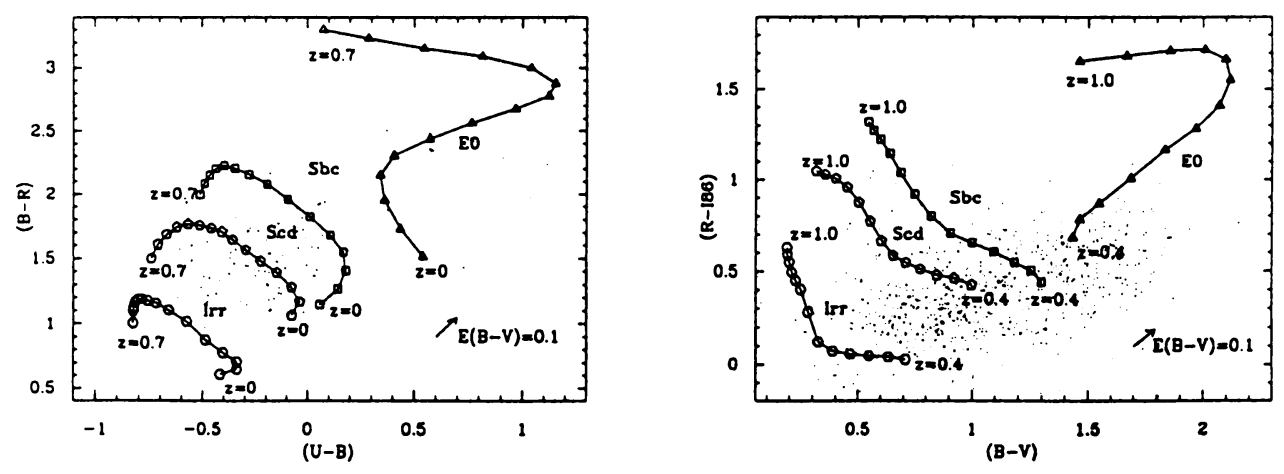

Fig. 1 Slices in multicolor space for blue (left) and red (right) colors in the Osmer et al. (1995) dataset, showing the color-vs.-redshift evolutionary tracks for typical E0, Sbc, Scd and Irr galaxies. A representative subsample of galaxies in the dataset are superposed. 